\title{
Analytical formulas for current dynamics and peak temperature during quench in MRI
}

\author{
Alex Protopopov \\ Correspondence: proto.alex@hotmail.com

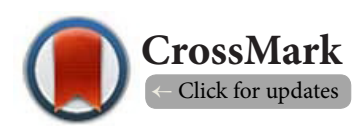

Moscow Institute of Physics and Technology, Russia.

\begin{abstract}
Quench of a superconducting magnet in MRI scanners may cause damage to the coil, but is unavoidable in some situations. Knowledge of the temporal evolution of current and the maximum temperature developed in the coil make it possible to determine the state the coil after the quench. Commonly, these parameters are computed using three-dimensional simulation programs. However, numerical models do not provide physical links between basic parameters of the magnet and quench dynamics. This paper presents analytical formulas that may serve as reasonable estimates, showing relationship between quench dynamics and parameters of a superconducting magnet.
\end{abstract}

Keywords: Superconducting coils, quench, magnet, MRI

\section{Introduction}

In recent years, great attention has been paid to the problem of simulation and assessment of aftermaths of quench in MRI. When quench occurs in a superconducting magnet, it is not always clear if the coil requires repairs or not. If it was damaged, then the magnet cannot be ramped up, and lengthy and expensive repairs are in order. However, if the probability of coil damage is minimal, then re-initialization is the optimal, cost-effective choice. Due to that, numerous cumbersome $3 \mathrm{D}$ simulation programmes have been created that enable the computation of quench dynamics in time and space, and allow estimation of values of current and peak temperature. Such programmes depend on many numerical parameters, which need to be known before the program can be executed. Sometimes, even little errors in the values of these parameters may lead to significant deviations. Therefore, a simple qualitative theory with relatively easily computable formulas, that can be used to estimate quench dynamics, would present an advantage in determining the state of the coil. In the present short communication such formulas are derived with very reasonable simplifications.

\section{Model}

In the simplified model, only lateral propagation of quench will be considered. Lateral propagation of quench is the spread of temperature increase along the superconducting wire. Thus, the effect that the rise of local temperature has on the surrounding wires is neglected. The reason for this assumption is the high velocity of quench propagation, about $2 \mathrm{~m} / \mathrm{s}$ for 240 A coils, and the design of the superconducting coil, in which each superconducting wire is separated from the neighboring wires by layers of supporting and shielding materials.

Figure 1 explains the model. Non-linear thermal dependence of the specific resistance $r$ around critical temperature $T_{c}$ is the principle phenomenon of the quench. What is essential, that it is practically zero below $T_{c}$, and abruptly rises to the nonzero value $r$ above this temperature. The exact temperature dependence of $r$ when $T>T_{c}$ is not important because it gives only second-order corrections. We shall assume, therefore,

$$
r=\left\{\begin{array}{ll}
0, & T<T_{\mathrm{c}} . \\
r, & T>T_{\mathrm{c}}
\end{array} .\right.
$$

This approximation may be called the bar-step approximation. Before the quench starts, the wire transmits initial current $I_{0}$. We assume that the quench originates at $x=x_{0}$ at the moment $t=0$. At this moment, specific resistance rises from zero to $r>0$, and electrical power

$$
\frac{r d x}{S} I^{2}
$$

begins to develop within this element of wire. Thus, variation of the heat during time increment $d t$ is

$$
d Q=\frac{r d x}{S} I^{2} \cdot d t
$$

As the temperature at $x=x$ rises due to this heat, heat flux spreads out of the volume $d V=S . d x$ due to temperature 


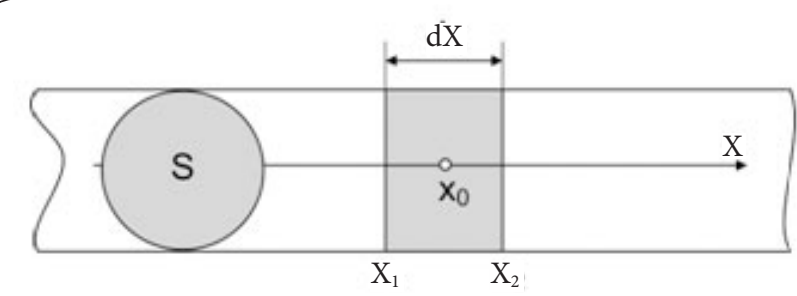

Figure 1. Superconducting wire with specific resistance [Ohm. $\mathrm{cm}$ ], cross section [cm2], thermal conductivity, and specific heat capacity.

gradient $d T / d x$. This leads to basic equation:

$$
\frac{r}{S^{2}} I^{2}+\frac{\partial}{\partial x}\left(k(x) \frac{\partial T}{\partial x}\right)=\rho C \frac{d T}{d t} .
$$

where $k(x)$ is thermal conductivity and $\rho$ - density of the superconducting wire. Note that the second term in the left-hand side acts negatively because the second derivative of temperature around $x_{0}$ is negative.

\section{Solution for the current}

Equation (4) has to be completed by the electrical equation, which follows from the energy conservation law: the sum of the magnetic energy $E$ stored in the magnet and the power transferred into heat $Q$ is constant:

$$
E+Q=\text { const } ;-d E=\delta Q
$$

Here $\delta Q$ is the total dissipation along the entire length of the superconducting wire:

$$
\delta Q=d t \cdot I^{2} \frac{1}{S} \int r(x) d x .
$$

According to phenomenology of quench, we introduce the longitudinal velocity of quench propagation $v_{0}[\mathrm{~cm} / \mathrm{s}]$. With it, the length $l$ of the wire section corrupted by quench is

$$
l=v_{0} t .
$$

Since other sections remain superconducting with $r=0$, the integral in the equation above is equal to $r \cdot v_{0} t$ :

$$
\delta Q=d t \cdot I^{2} \frac{r \cdot v_{0}}{S} t
$$

Magnetic energy is

$$
E=\frac{1}{2} L I^{2},
$$

where $L$ is the magnet inductance, and

$$
d E=L \cdot I d I \text {, }
$$

which makes the equation

$$
-L I d I=I^{2} \frac{r \cdot v_{0}}{S} t d t .
$$

It gives simple analytical formula for evolution of the current during quench:

$$
I=I_{0} e^{-(t / \tau)^{2},} \tau=\sqrt{\frac{2 S L}{r v_{0}}} .
$$

We introduced parameter $\tau$ that may be called the quench time-the time interval during which the current drops $e$ times from the value of stable operation. The resistance per unit length

$$
\eta=\frac{r}{S}[\mathrm{Ohm} / \mathrm{m}]
$$

is always known: it is one of the design parameters of the magnet wire that is always carefully characterized. Typically, $\eta \approx 0.15 \mathrm{Ohm} / \mathrm{m}$ [1-4]. With this, the quench time transforms to

$$
\tau=\sqrt{\frac{2 L}{\eta v_{0}}} .
$$

Dimension of the right-hand side is time since inductance $L$ measured in Henry is equal to

$$
\mathrm{H}=\frac{\mathrm{m}^{2} \mathrm{~kg}}{\mathrm{C}^{2}}=\frac{\mathrm{m}^{2} \mathrm{~kg}}{\mathrm{~s}^{2} \mathrm{~A}^{2}}=\frac{\mathrm{J}}{\mathrm{A}^{2}}=\frac{\mathrm{Wb}}{\mathrm{A}}=\frac{\mathrm{V} \cdot \mathrm{s}}{\mathrm{A}}=\frac{\mathrm{s}^{2}}{\mathrm{~F}}=\Omega \cdot \mathrm{s},
$$

where $\mathrm{C}$ stands for the Coulomb, Wb - for the Weber (magnetic flux), F - for the Farad, W - for the Ohm. Formula (14) for the quench time clearly shows that the smaller the magnet inductance the shorter the quench. Alternatively, the smaller the resistance above superconducting threshold the longer the quench.

Now, we shall compare the formula for the current (12) with experimental results. Qualitatively, it is consistent with all the experimental results on quench published so far. For instance, Figure 2 shows the Gaussian curve (12) fitted into the results published in [5], with the fitting parameters $I_{0}$ $=200 \mathrm{~A}$ and $\tau=7 \mathrm{~s}$.

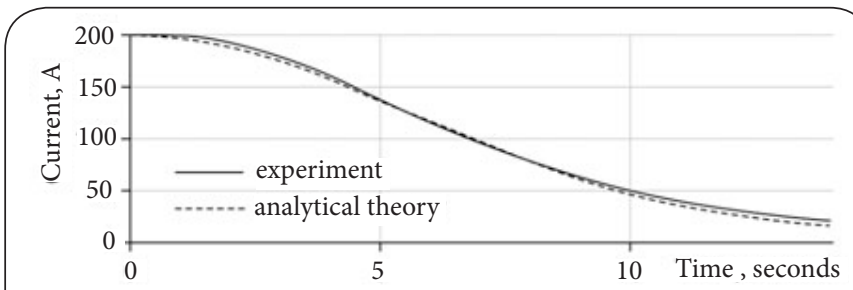

Figure 2. Comparison of experimental quench current [5] with analytical formula (12).

Whatever good the qualitative agreement is, does our theory 
describe the phenomenon quantitatively? The answer to this question can only be given with the complete set of data, characterizing the superconducting magnet. One of the few publications that provide necessary data is [6]. Technical specifications of the magnet described in this article are reproduced in the Table 1 below.

Table 1. Parameters of the superconducting magnet [6].

\begin{tabular}{|c|c|c|c|}
\hline \multicolumn{4}{|l|}{ Parameters } \\
\hline Dipole magnetic field & $1.6 \mathrm{~T}$ & Average turn length & $6.576 \mathrm{~m}$ \\
\hline Usable aperture & $0.38 \mathrm{~m} \times 0.14 \mathrm{~m}$ & Nominal current & $232 \mathrm{~A}$ \\
\hline $\mathrm{N}$ of layers $\times \mathrm{n}$ of turns & $20 \times 28=560$ & Stored energy & $414 \mathrm{~kJ}$ \\
\hline \multicolumn{2}{|c|}{ Superconducting material $\mathrm{NbTi}$} & NbTi cross section & $0.173 \mathrm{~mm}^{2}$ \\
\hline Cladding & $\mathrm{Cu}$ & Copper cross section & $1.862 \mathrm{~mm}^{2}$ \\
\hline
\end{tabular}

It uses $\mathrm{NbTi}$ superconducting filaments evenly spread within the $\mathrm{Nb}$ wire, which is embedded into a bulk cupper strip.

Figure $\mathbf{3}$ compares experimental data for quench current from [6] with our theory. Analytical formula (12) is fitted into the experimental data with the value $\tau=12 \mathrm{~s}$. The immediate conclusion that follows from this figure is that, qualitatively, the analytical formula is again in agreement with the experiment. Next, consider quantitative details, specifically the quench velocity $v_{0}$. From (12) it follows that

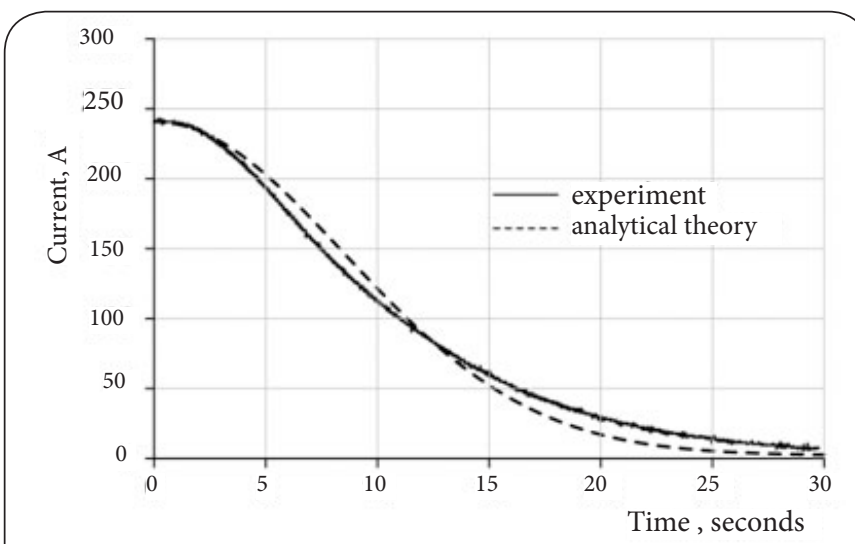

Figure 3. Comparison of experimental quench current [6] with analytical formula (12).

$$
v_{0}=\frac{2 L}{\eta \tau^{2}}
$$

The table gives the value of magnetic energy stored in the magnet

$$
E=\frac{1}{2} L I^{2}=414 \mathrm{~kJ}
$$

and the current $I=232 \mathrm{~A}$. This is the single-coil magnet, so that we find $L=15.4 \mathrm{H}$. For $\eta$ we do not have necessary data, but we may take the typical value cited above: $\eta=0.15 \mathrm{Ohm} / \mathrm{m}$.
With all these data, formula (15) gives $v_{0}=1.4 \mathrm{~m} / \mathrm{s}$. Quench velocity can hardly be measured, but luckily, we can compare this value to the value numerically simulated in [6] and presented in Figure 4 in the form of lines. Our value, computed for the particular current $232 \mathrm{~A}$, is shown by the open circle. Thus, analytical formula (15) gives the result consistent with

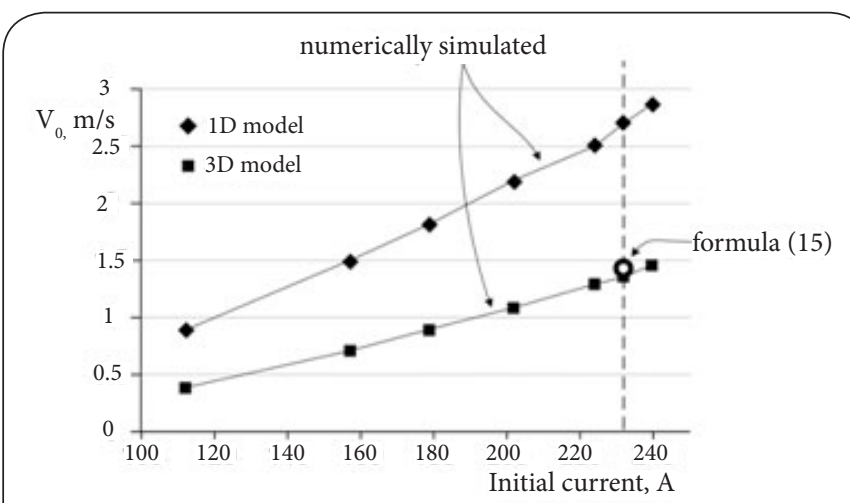

Figure 4. Quench velocity simulated by numerical models [6] and the value given by analytical formula (15).

the numerical models.

\section{Solution for the temperature}

Quench current and propagation velocity are, of course, important parameters, but even more important is evaluation of the maximum spot temperature that is developed during quench. If this temperature exceeds critical value, then the superconducting coil is probably damaged. Consider the basic equation (4) again. In it, specific heat capacity $C$ is a complicated function of temperature. Copper is the main heat absorbing material of the wire, and for it $C(T)$ is shown in Figure 5 [4]. With temperature being in the interval of $4 \mathrm{~K}<T<100 \mathrm{~K}$, specific heat capacity of copper can be reasonably described by a second-order approximation $C=0.03 . T^{2}$, or in general:

$$
C=\alpha T^{\beta-1} \text {. }
$$

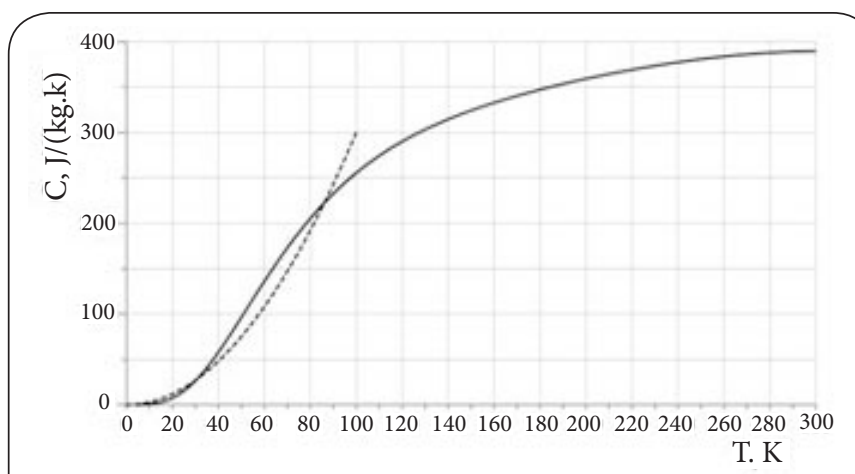

Figure 5. Copper specific heat (solid line) and its approximation $C=0.03 T^{2}$ (dashed line). 
In such an approximation, dimension of $\alpha$ is $\mathrm{J} /\left(\mathrm{kg} \times \mathrm{K}^{\mathrm{b}}\right)$ and $\beta$ is dimensionless.

The basic equation (4) is the partial differential equation of the second order that cannot be solved analytically without simplifying assumptions. First, assume thermal conductivity $k$ independent of the temperature, and as such, independent of $x$. This assumption is supported by experimental phenomenology, showing that around the critical temperature $T_{\mathrm{c}}, k$ varies insignificantly [8].

Our main goal is to derive the formula for the maximum spot temperature. This point is located at the quench origin $x_{0}=0$, from which the quench wave spreads to the more distant sections of the wire. Here, the term with thermal conductivity $k$, describing heat transfer from more heated areas of the wire to the less heated ones, is insignificant and may be dropped. Indeed, after the wave of high temperature has started moving, the temperature of the adjacent regions immediately to the right and to the left from $x_{0}$ becomes almost the same as the temperature at $x_{0}$, which means that the temperature gradient in $x_{0}$ is close to zero. As such, the second derivative at $x=x_{0}$ may be neglected.

This leads us to the equation

$$
\eta I^{2} d t=\mu C d T
$$

where again $\eta=r / S$ is the resistance of the wire per unit length, and $\mu=\rho \cdot S$ is the mass of the wire per unit length. Substituting (12) and (16) and integrating over temperature, we obtain:

$$
\frac{\eta \tau I_{0}^{2}}{2} \sqrt{\frac{\pi}{2}} \operatorname{erf}\left(\frac{t}{\tau} \sqrt{2}\right)=\mu p\left(T^{\beta}-T_{0}^{\beta}\right),
$$

where $T_{0}=4.2 \mathrm{~K}$ is the initial temperature for the superconducting wire. This gives the solution:

$$
T=\left[\frac{\eta \tau I_{0}^{2}}{2 \mu p} \sqrt{\frac{\pi}{2}} \operatorname{erf}\left(\frac{t}{\tau} \sqrt{2}\right)+T_{0}^{\beta}\right]^{1 / \beta}
$$

Here $\operatorname{erf}(x)=\frac{2}{\sqrt{\pi}} \int_{0}^{x} e^{-y^{2}} d y$

is the well-known error-function, and

$$
p=\frac{\alpha}{\beta}
$$

is an important parameter of a superconducting wire, determining dynamics of the temperature. Dimension of this parameter is the same as $\alpha$, i.e., $\mathrm{J} /\left(\mathrm{kg} \times \mathrm{K}^{\mathrm{b}}\right)$.

With $\mathrm{t} \rightarrow \infty$, temperature stabilizes at the maximum value $T_{\text {max }}$. Since $\operatorname{erf}(\infty)=1$ and $T_{\text {max }}>T_{0}$

$$
T_{\max } \approx\left[\frac{\eta \tau I_{0}^{2}}{2 \mu p} \sqrt{\frac{\pi}{2}}\right]^{1 / \beta}
$$

This is the very important result, showing how the maximum temperature depends on the parameters of the super-conducting wire, current, and quench time. It is not obvious that the dimension of this result is equal to Kelvin, therefore we show this explicitly:

$$
\frac{\left[\frac{\Omega}{\mathrm{m}}\right][s]\left[A^{2}\right]}{\left[\frac{\mathrm{kg}}{\mathrm{m}}\right]\left[\frac{\mathrm{J}}{\mathrm{kg} \cdot \mathrm{K}^{\beta}}\right]}=\frac{\left[\frac{\mathrm{J}}{A^{2}}\right]\left[A^{2}\right]}{J} K^{\beta}=K^{\beta} .
$$

Compare (21) to the results, computed in [6], using the 3D model (Figure 6). High temperature specific resistance of a superconducting material itself is very high. For instance, for NbTi $r \sim 5 \cdot 10^{-7} \mathrm{Ohm} \cdot \mathrm{m}$ at $T \sim 100 \mathrm{~K}$, whereas for the copper cladding $r \sim 10^{-10} \mathrm{Ohm} \cdot \mathrm{m}$ [4]. Therefore, high-resistance section of the superconducting filament will be bypassed by surrounding copper, so that the values for $\eta$ and $\mu$ in (21) should be those for the copper. We used the following set of parameters, appropriate to the result described in [6]:

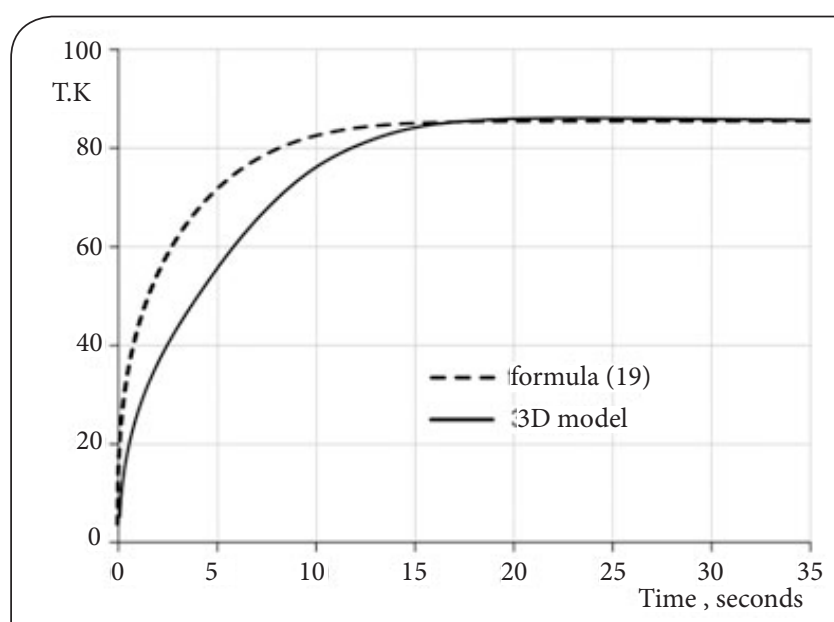

Figure 6. Evolution of quench spot temperature. The 3D model is computed in [6].

$I_{0}=232 \mathrm{~A} ;$

$r=1.0 \cdot 10^{-10} \mathrm{Ohm} \cdot \mathrm{m}$ - specific resistance of copper at

$T \sim 100 \mathrm{~K}$;

$S=1.862 \mathrm{~mm}^{2}$ - cross section of a copper strip;

$\eta=r / S=5.4 \cdot 10^{-5} \mathrm{Ohm} / \mathrm{m}$;

$\tau=12 \mathrm{~s}-$ as determined from Figure 3 ;

$\rho=8.96 \cdot 10^{3} \mathrm{~kg} / \mathrm{m}^{3}$ - density of copper;

$\mu=\rho S=0.0167 \mathrm{~kg} / \mathrm{m}$ for copper strip;

$\alpha=0.03 \mathrm{~J} /\left(\mathrm{kg} \times \mathrm{K}^{3}\right)$ as in Figure 5;

$\beta=3$;

$\mathrm{T}_{0}=4.2 \mathrm{~K}$.

Direct substitution of the above parameters into (21) gives 
$T_{\max }=51 \mathrm{~K}$, which is not very far from the experimental value $T_{\max } \approx 85 \mathrm{~K}$ [6]. The largest part of uncertainty lies in the ratio $\eta / \mu$. The above values of $\eta$ and $\mu$ give $\eta / \mu$ $=0.003$ in SI units. Correcting it to 0.015 , we obtain necessary $T_{\max }=85 \mathrm{~K}$. With this only correction, theoretical curve of formula (19) is shown in Figure 6.

Although entire qualitative behavior of the analytical formula preserves the essential features of the spot temperature dynamics, like steep initial trend and smooth finish to a stationary value of $T_{\max }$, it differs from numerical computations in the middle part of the interval. Nonetheless, analytical formulas (19)-(21) combine all the parameters together, explicitly showing the influence of each of them - a feature that cannot be obtained by numerical computations.

\section{Conclusion}

Analytical formula derived for temporal evolution of the quench current is in good quantitative agreement with experimental data. This formula establishes relation between the quench time and very few basic parameters of the superconducting magnet: wire resistance per unit length, magnet inductance, and longitudinal quench speed. The strength of this solution is not the simplicity of the Gaussian function that describes evolution of the current, but that it establishes quantitatively correct general analytical relationship between the current and only three basic measurable parameters of the superconducting magnet.

Using the formula for the quench current, longitudinal quench velocity can be estimated from the recorded log-file of the quench event. For that, the Gaussian function should be fitted into the experimental curve with only one fitting parameter: the quench time. Quench velocity turned out to be inversely proportional to the quench time squared.

Analytical formula for temperature dynamics is in qualitative agreement with experimental data. From it, the maximum value of the temperature increase during quench can be estimated. The estimate of the maximum temperature determines probability of whether or not the superconducting coil has been damaged during quench. This very initial assessment, done quickly and without applying cumbersome numerical computations, may be important for making initial decision after quench has happened.

The overall conclusion is that even very simple analytical analysis, accounting for only few driving physical considerations, may provide valuable initial insight into rather complicated problem.

\section{Competing interests}

The author declares that he has no competing interests.

Publication history

EIC: Robert A. Lodder, University of Kentucky, USA.

Domenico Rubello, Padova University, Italy.

Received: 06-Feb-2014 Final Revised: 17-May-2014

Accepted: 17-Jul-2014 Published: 24-Jul-2014

\section{References}

1. H.-I. Du, M.-J. Kim and Y.-J. Kim. Study on resistance characteristics and operating conditions of YBCO thin-film wire for current limitation considering insulation layer. Physica C: Superconductivity. 2010; 470:1666-1670. | Article

2. G.-J. Lee. Superconductivity Application in Power Systems. in Applications of High-T superconductivity, Ed. A.M. Luiz, Chapter 3. In Tech. 2011; 260. | Article

3. L. Xiaohang. Superconductivity Devices in Wind Farm. in Wind Energy Management, Ed. P. Bhattacharya, Chapter 5. In Tech. 2011; 128. I Pdf

4. G. Manfreda. Review of ROXIE's Material Properties Data Base for Quench Simulation. TE Technology department internal note. 2011; 35. I Pdf

5. A. Stenvall, N. Magnusson and Z. Jelinek. Electromagnetic viewpoints

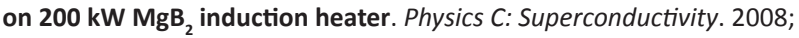
468:487-491. | Article

6. P. Szwangruber, F. Toral and E. Floch. Quench calculations and measurements on the FAIR Supr-FRS dipole. Physics Procedia. 2011; 1-6. | Article

7. S. Russenschuck. Material Property Data for Quench Simulations. Appendix 1. in: Field Computation for Accelerator Magnets: Analytical and Numerical Methods for Electromagnetic Design and optimization, Wiley-VCH Verlag GmbH\&Co, KGaA, Weinheim, Germany. 2011; 703-715. I Pdf

8. T.P. Sheahen. Introduction to high-temperature superconductivity. Plenum Press, N-Y. 1994; 371. | Book

\section{Citation:}

Protopopov A. Analytical formulas for current dynamics and peak temperature during quench in MRI. Med Instrum. 2014; 2:3.

http://dx.doi.org/10.7243/2052-6962-2-3 